\title{
Autonomic cardiac control in animal models of cardiovascular diseases II. Variability analysis in transgenic rats with $\alpha$-tropomyosin mutations Asp175Asn and Glu180Gly
}

\author{
Dirk Wernicke ${ }^{1, a, *}$, Niels Wessel ${ }^{2, a}$, Hagen \\ Malberg ${ }^{3}$, Ralph Plehm ${ }^{1}$, Robert Bauernschmitt ${ }^{4}$ \\ and Ludwig Thierfelder ${ }^{1}$ \\ ${ }^{1}$ Max Delbrück Center for Molecular Medicine, Berlin, \\ Germany \\ 2 University of Potsdam, Department of Physics, \\ Potsdam, Germany \\ ${ }^{3}$ University of Karlsruhe, Institute for Applied Computer \\ Science/Automation, Karlsruhe, Germany \\ ${ }^{4}$ German Heart Center Munich, Clinic for \\ Cardiovascular Surgery, Munich, Germany
}

\begin{abstract}
Animal models of cardiovascular diseases allow to investigate relevant pathogenetic mechanisms in detail. In the present study, the mutations Asp175Asn and Glu180Gly in $\alpha$-tropomyosin (TPM1), known cause familiar hypertrophic cardiomyopathy (FHC) were studied for changes in hemodynamic parameters and spontaneous baroreflex regulation in transgenic rats in comparison to transgenic and non-transgenic controls by telemetry. Heart rate variability (HRV) and blood pressure variability (BPV) were analyzed using time- and frequency domain, as well as non-linear measures. The dual sequence method was used for the estimation of the baroreflex regulation. In transgenic rats harboring mutated TPM1, changes in HRV were detected during exercise, but not at rest. Both mutations, Asp175Asn and Glu180Gly, caused increased low frequency power. In addition, in animals with mutation Asp175Asn a reduced total HRV was observed. BPV did not show any differences between all transgenic animal lines. During exercise, a strong increase in the number of bradycardic and tachycardic fluctuations accompanied with decreased baroreflex sensitivity (BRS) was detected in animals with either TPM1 mutation, Asp175Asn or Glu180Gly. These data suggest, that the analysis of cardiac autonomic control, particularly of baroreflex regulation, represents a powerful non-invasive approach to investigate the effects of subtle changes in sarcomeric architecture on cardiac physiology in vivo. In case of mutations Asp175Asn or Glu180Gly in TPM1, early detection of alterations in autonomic cardiac control
\end{abstract}

\footnotetext{
a These authors contributed equally to this article.

*Corresponding author: Dirk Wernicke, Max-Delbrück-Center for Molecular Medicine, Robert-Roessle-Str. 10, 13092 Berlin, Germany

Phone: +49-30-9406 3707

Fax: +49-30-94 94161

E-mail: dwernic@mdc-berlin.de
}

could help to prevent sudden cardiac death in affected persons.

Keywords: baroreflex sensitivity; blood pressure variability; familiar hypertrophic cardiomyopathy; heart rate variability.

\section{Introduction}

During the last decade, substantial efforts were made to discover the genetic reasons of cardiovascular diseases. Based on this genetic information, subsequent studies can now be initiated to investigate the pathogenetic effects of disease-causing mutations on cardiac physiology. In this context, advanced technologies in biosignal processing provide enhanced methods to study cardiac function in vivo in a non-invasive way. In particular, their application in studies of cardiovascular diseases in animal models represents a useful approach for getting insights into relevant pathogenetic mechanisms, which will be elucidated in this Part II of a bipartite series on this general topic. A detailed description of the biosignal processing tools was given by Part I [23].

Based on the conflicting results on autonomic nervous control in patients with familiar hypertrophic cardiomyopathy $(\mathrm{FHC})[5,7,21]$, transgenic rats overexpressing either mutation Asp175Asn or Glu180Gly in TPM1 were investigated for the hemodynamic effects on heart rate, aortic blood pressure, and spontaneous baroreflex regulation caused by these two missense mutations. The transgenic rat strains used in the present research were established to study the effect of low-level overexpression of human $\alpha$-tropomyosin (TPM1), harboring mutation Asp175Asn or Glu180Gly, on cardiac physiology in vitro as well as in vivo [22]. So, autonomic cardiac control in these conditions could be investigated in those transgenic animals at rest as well as during exercise, including the periods before and after completing the exercise protocol in running wheels.

\section{Materials and methods}

The various analytical methods for heart rate variability (HRV), blood pressure variability (BPV), and baroreflex sensitivity (BRS) analyses are described in Part I [23] of this bipartite report on autonomic cardiac control in animal models of cardiovascular diseases. Therefore, this section now supplements the details on the animal model. 


\section{Animal care and exercise}

The transgenic rats were prepared as previously described [22]. Six animals of each transgenic line and six non-transgenic controls underwent the training procedure in "passive" running wheels which were driven by an electric motor. In parallel, six animals of each transgenic line and six non-transgenic control animals were analyzed at rest. The telemetry and data acquisition system equipped with Dataquest LabPRO software system was used as suggested by the manufacturer (Data Sciences Inc., St. Paul, MN, USA). The system and the sensor implantation procedure are described by Brockway et al. [4].

\section{Statistics}

Group summaries are expressed as mean value \pm standard error. The non-parametric Kruskal-Wallis test was used to detect intergroup differences, whereas differences between two groups were analyzed using Mann-Whitney U-test. In all tests, the criterion for statistical significance was Bonferroni-Holm corrected $p<0.05$.

\section{Results}

HRV, BPV, and spontaneous baroreflex activity were analyzed in transgenic rat lines, overexpressing either the human wild-type (WT) TPM1 (line A (WT)), or TPM1 with mutation Asp175Asn [line B (Asp175Asn)], or with mutation Glu180Gly [line C (Glu180Gly)]; time domain and frequency domain parameters, as well as non-linear measures as described in Part I [23] were determined.
Hemodynamic parameters were detected at rest and during exercise at the beginning as well as after completing an exercise protocol in running wheels.

No differences were seen in HRV between sedentary animals of all three transgenic lines A (WT), B (Asp175Asn), and C (Glu180Gly) at the beginning of the training procedure as well as after completing the exercise protocol. At the end of the exercise protocol, however, changes in HRV were observed in animals of both mutant lines, B (Asp175Asn) and C (Glu180Gly). As shown in Table 1B, reduced total HRV was observed in line B (Asp175Asn) compared to line C (Glu180Gly) and the transgenic control line A (WT) based on the determination of the root mean square of successive differences (RMSSD) and the intermittently decreased variability POLVAR3, one time domain and one symbolic dynamics parameter, respectively. At the same time, a 1.8-fold increase in the low/high frequency component ratio (LF/HF) was detected in line $B$ (Asp175Asn) compared to line A (WT). In comparison, line C (Glu180Gly) is characterized by a 10-fold elevation of the low frequency component (LF) and a 3.8-fold increase in the LF/ $\mathrm{HF}$ ratio. These changes in HRV parameters at the end of the exercise protocol observed in both mutant lines, B (Asp175Asn) and C (Glu180Gly), reached levels of significance (Table 1B), but were either already detected or somehow indicated to rise at the beginning of the exercise protocol (Table 1A). Figures 1-3, showing representative $1 \mathrm{~h}$ recording strips of beat-to-beat intervals for animals of line A (WT), B (Asp175Asn), and C (Glu180Gly) during exercise near the end of the protocol, illustrate the reduced total HRV in line $B$ (Asp175Asn) and the increased LF component of HRV in line C (Glu180Gly).

Table 1 HRV parameters during exercise.

(A) HRV parameters at the beginning of the training protocol.

\begin{tabular}{lcccc}
\hline & $\mathrm{A}(\mathrm{WT})$ & $\mathrm{B}$ (Asp175Asn) & $\mathrm{C}$ (Glu180Gly) & $\mathrm{P}_{\mathrm{Kw}}$ \\
\hline MEANNN & $133.22 \pm 3.33$ & $139.94 \pm 2.62$ & $144.82 \pm 3.98$ & $\mathrm{n} . \mathrm{s}$. \\
SDNN & $6.59 \pm 0.84$ & $6.35 \pm 0.13$ & $6.94 \pm 0.42$ & $\mathrm{n} . \mathrm{s}$. \\
RMSSD & $3.72 \pm 0.09^{*}$ & $2.86 \pm 0.14 \ddagger$ & $4.00 \pm 0.21$ & $<0.01$ \\
LF & $0.0012 \pm 0.0002$ & $0.0011 \pm 0.0004 \ddagger$ & $0.0038 \pm 0.0008 \dagger$ & $<0.01$ \\
HF & $0.0008 \pm 0.0001$ & $0.0006 \pm 0.0002 \ddagger$ & $0.0015 \pm 0.0002 \dagger$ & $<0.01$ \\
LF/HF & $1.49 \pm 0.18$ & $1.71 \pm 0.16$ & $2.57 \pm 0.49$ & n.s. \\
POLVAR3 & $0.04 \pm 0.01^{*}$ & $0.43 \pm 0.06 \ddagger$ & $0.15 \pm 0.03 \dagger$ & $<0.01$ \\
\hline
\end{tabular}

(B) HRV parameters at the end of the training protocol.

\begin{tabular}{lcccc}
\hline & $\mathrm{A}(\mathrm{WT})$ & $\mathrm{B}$ (Asp175Asn) & C (Glu180Gly) & $P_{\mathrm{KW}}$ \\
\hline MEANNN & $133.43 \pm 3.93$ & $138.33 \pm 2.60$ & $151.63 \pm 6.19 \dagger$ & $<0.05$ \\
SDNN & $6.56 \pm 1.27$ & $5.49 \pm 0.30 \ddagger$ & $9.31 \pm 0.85 \S$ & $<0.05$ \\
RMSSD & $4.44 \pm 0.46^{*}$ & $2.96 \pm 0.23 \ddagger$ & $5.21 \pm 0.45$ & $<0.01$ \\
LF & $0.0008 \pm 0.0001$ & $0.0012 \pm 0.0003 \ddagger$ & $0.008 \pm 0.002 \dagger \S$ & $<0.01$ \\
HF & $0.0009 \pm 0.0001$ & $0.0008 \pm 0.0002 \ddagger$ & $0.002 \pm 0.001 \dagger$ & $<0.01$ \\
LF/HF & $0.92 \pm 0.08^{\star} \S$ & $1.63 \pm 0.19 \ddagger$ & $3.49 \pm 0.69 \dagger$ & $<0.001$ \\
POLVAR3 & $0.05 \pm 0.01^{*}$ & $0.44 \pm 0.03 \ddagger$ & $0.10 \pm 0.05$ & $<0.01$ \\
\hline
\end{tabular}

HRV parameters of transgenic animals were calculated from $1 \mathrm{~h}$ recordings during exercise. See [23] for detailed description of the parameters.

${ }^{*} p<0.05$ A (WT) vs. B (Asp175Asn).

† $p<0.05$ A (WT) vs. C (Glu180Gly).

$\ddagger \mathrm{p}<0.05 \mathrm{~B}$ (Asp175Asn) vs. C (Glu180Gly).

$\S p<0.05$ begin vs. end of training.

Mean values were calculated from 6 animals. 

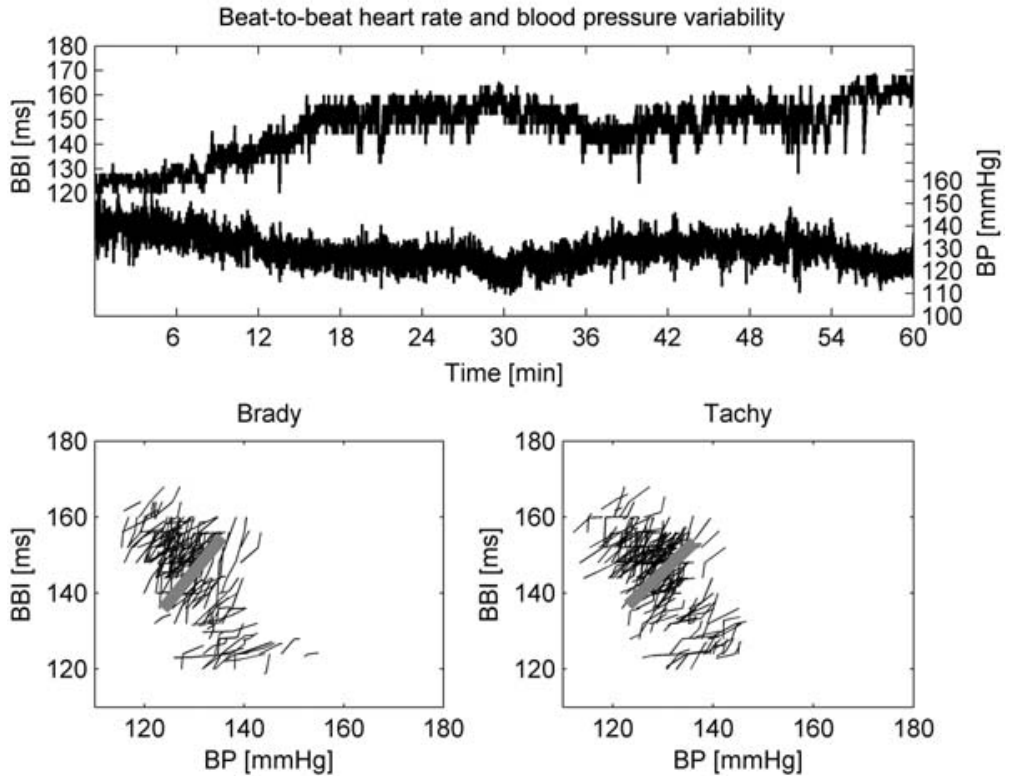

Figure 1 Beat-to-beat heart rate and blood pressure recording of line A (WT).

One hour recording strip of beat-to-beat (BB) intervals and blood pressure values (BP) of a rat of line A (WT) during exercise at the end of the training procedure are shown on the top. Below, the patterns of the corresponding bradycardic (bottom left) and tachycardic (bottom right) baroreflex fluctuations are demonstrated. Bars represent average bradycardic/tachycardic BRS.

The investigation of the blood pressure $(\mathrm{BP})$ regulation revealed no differences in mean $\mathrm{BP}$ values and in BPV parameters between animals of all three transgenic lines A (WT), B (Asp175Asn), and C (Glu180Gly) at rest and during exercise, as demonstrated here for representative $\mathrm{BP}$ recordings of animals under physical load (Figures 1-3).

The analysis of the spontaneous baroreflex activity showed a strong influence of the baroreflex on BP regulation in animals of both mutant lines, B (Asp175Asn) and C (Glu180Gly), compared to transgenic controls. In line C (Glu180Gly), the number of bradycardic and tachycardic baroreflex events, estimated using the dual sequence method [23], was increased 7-fold and 9-fold, respectively. In line B (Asp175Asn), the number of bradycardic and tachycardic events was elevated 2.5-fold and 3-fold, respectively (see also Figures 1-3, bottom). The comparison of baroreflex indices before and after the physical training protocol in running revealed a more pronounced positive training effect on baroreflex regulation in transgenic controls compared to both mutant transgenic lines: While the sensitivity of baroreflex regulation remained unchanged in all three transgenic rat lines, the number of bradycardic and tachycardic events was elevated 2 -fold in line A (WT), but only by about $25 \%$ in lines B (Asp175Asn) and C (Glu180Gly) (Table 2).
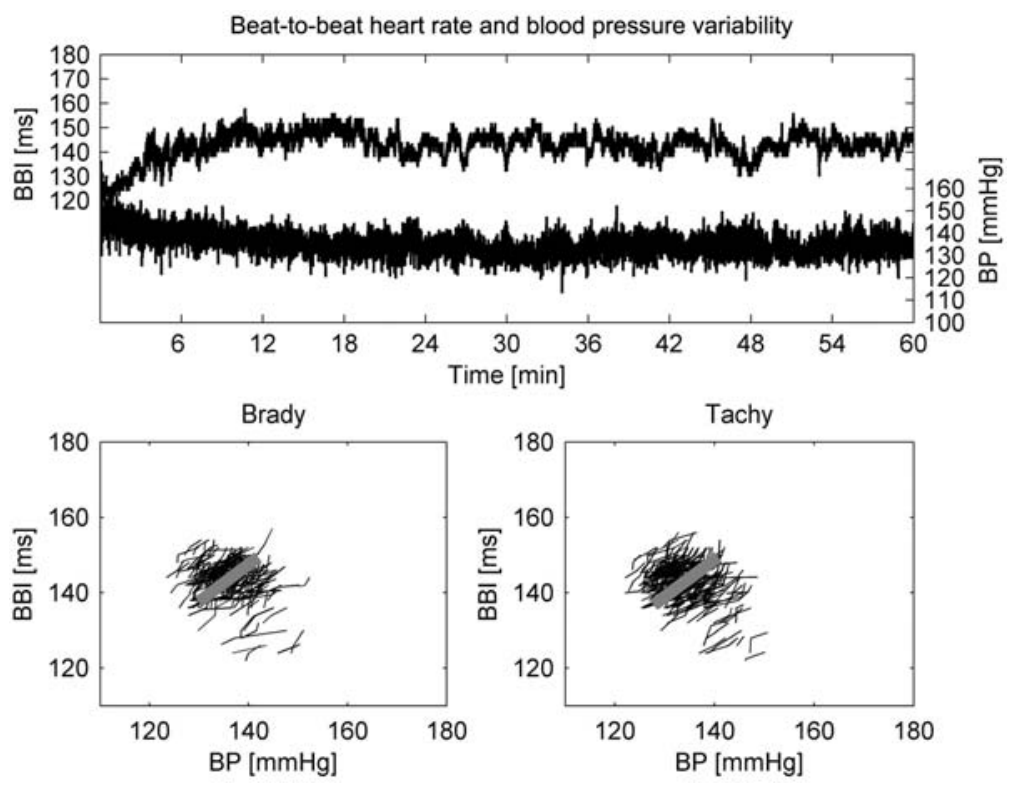

Figure 2 Beat-to-beat heart rate and blood pressure recording of line B (Asp175Asn). Same as Figure 1 but now for a rat of line B (Asp175Asn). 

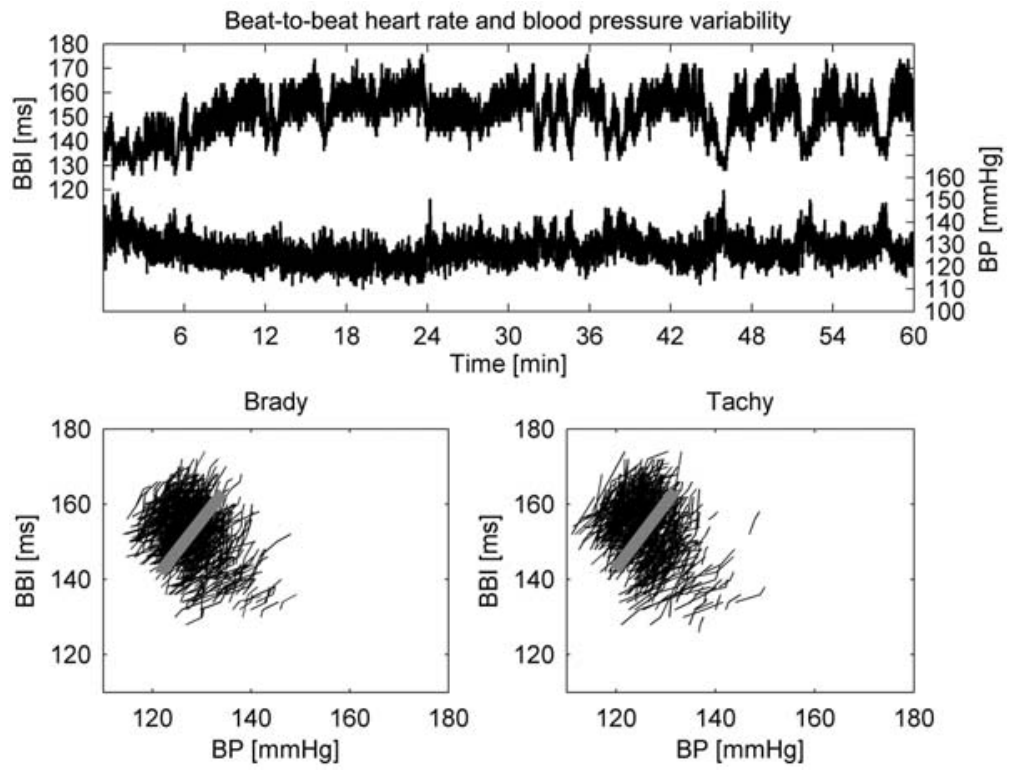

Figure 3 Beat-to-beat heart rate and blood pressure recording of line C (Glu180Gly).

Same as Figure 1 but now for a rat of line C (Glu180Gly).

Table 2 Spontaneous baroreflex parameters during exercise.

(A) Spontaneous baroreflex parameters at the beginning of the training protocol.

\begin{tabular}{llccl}
\hline & A (WT) & B (Asp175Asn) & C (Glu180Gly) & $P_{\text {KW }}$ \\
\hline Brady events & $81.75 \pm 14.17$ & $211.20 \pm 26.16 \dagger$ & $565.5 \pm 78.12^{*}$ & $<0.001$ \\
Tachy events & $60.75 \pm 8.77$ & $186.20 \pm 20.03 \dagger$ & $571.7 \pm 86.17^{\star}$ & $<0.001$ \\
Brady slope & $5.03 \pm 1.26$ & $3.54 \pm 0.60$ & $2.82 \pm 0.24$ & n.s. \\
Tachy slope & $4.32 \pm 1.14$ & $2.47 \pm 0.16$ & $2.38 \pm 0.09^{\star}$ & $<0.01$ \\
\hline
\end{tabular}

(B) Spontaneous baroreflex parameters at the end of the training protocol.

\begin{tabular}{lcccc}
\hline & A (WT) & B (Asp175Asn) & C (Glu180Gly) & $P_{\mathrm{kw}}$ \\
\hline Brady events & $159.25 \pm 26.60 \ddagger$ & $243.00 \pm 26.12 \dagger$ & $718.83 \pm 106.28^{*}$ & $<0.001$ \\
Tachy events & $114.25 \pm 17.08 \ddagger$ & $242.66 \pm 24.66 \dagger$ & $673.66 \pm 55.83^{*}$ & $<0.001$ \\
Brady slope & $4.55 \pm 0.99$ & $2.95 \pm 0.37$ & $3.09 \pm 0.48^{*}$ & $<0.01$ \\
Tachy slope & $5.54 \pm 1.34$ & $2.41 \pm 0.50$ & $2.36 \pm 0.16^{*}$ & $<0.01$ \\
\hline
\end{tabular}

Spontaneous baroreflex parameters of transgenic animals were calculated from $1 \mathrm{~h}$ recordings during exercise. "Brady events" stands for the number of all bradycardic baroreflex events, "tachy events" for the number of all tachycardic baroreflex events, "brady slope" for the average bradycardic slope, and "tachy slope" for the average tachycardic slope. For detailed description of these parameters, see [23].

${ }^{*} p<0.05 \mathrm{~A}$ (WT) vs. C (Glu180Gly).

$\dagger p<0.05$ B (Asp175Asn) vs. C (Glu180Gly).

$\ddagger p<0.05$ begin vs. end of training.

Mean values were calculated from 6 animals.

Analyzing the baroreflex regulation in resting animals, a reduced BRS was detected in line B (Asp175Asn) compared to line C (Glu180Gly) shown by a decreased average bradycardic slope (Table $3 \mathrm{~A})$. In this animal line, reduced BRS is compensated by an increased number of bradycardic events. After completing the training protocol, in animals of line B (Asp175Asn) baroreflex regulation recovered as indicated by an increase in the average bradycardic slope accompanied with the normalization of the number of bradycardic events (Table 3B). At the same time, in line B (Asp175Asn) the baroreflex remained impaired as demonstrated by a strong increase in the number of tachycardic events (Table 3B). In comparison, in line C (Glu180Gly) baroreflex regulation was not affected by physical training. In line A (WT), a reduced number of bradycardic events accompanied with an unchanged average bradycardic slope reflects a positive training effect in these control animals (Table 3).

In all transgenic animals, the number of bradycardic and tachycardic events was markedly increased in animals at rest in comparison to animals under physical load (Tables 2 and 3). At the same time, only in transgenic control animals of line A (WT) a strong increase in BRS was observed during exercise compared to rest. This increase in BRS is most likely based on the generally elevated BPV in all transgenic rat lines during exercise compared to rest (data not shown).

There were no significant differences in HRV, BPV, and BRS between the transgenic line A(WT) and non-transgenic controls (data not shown). 
Table 3 Spontaneous baroreflex parameters at rest.

(A) Spontaneous baroreflex parameters before starting the training protocol.

\begin{tabular}{lccc}
\hline & A (WT) & B (Asp175Asn) & C (Glu180Gly) \\
\hline Brady events & $1938.5 \pm 289.9$ & $2453.2 \pm 619.8$ & $1978.6 \pm 295.7$ \\
Tachy events & $1900.5 \pm 258.4$ & $1797.6 \pm 480.3$ & $1848.0 \pm 250.6$ \\
Brady slope & $1.96 \pm 0.17$ & $1.69 \pm 0.24 \dagger$ & $2.40 \pm 0.45$ \\
Tachy slope & $2.07 \pm 0.11$ & $2.23 \pm 0.31$ & n.s. \\
\hline
\end{tabular}

(B) Spontaneous baroreflex parameters after completing the training protocol.

\begin{tabular}{lccc}
\hline & A (WT) & B (Asp175Asn) & C (Glu180Gly) \\
\hline Brady events & $1325.0 \pm 193.8 \ddagger$ & $1978.6 \pm 414.1 \ddagger$ & $1924.5 \pm 230.4^{*}$ \\
Tachy events & $1707.3 \pm 344.2$ & $2853.4 \pm 590.0 \ddagger$ & $1971.5 \pm 186.6^{*}$ \\
Brady slope & $2.06 \pm 0.27$ & $2.21 \pm 0.26$ & $2.46 \pm 0.16^{*}$ \\
Tachy slope & $1.69 \pm 0.12$ & $1.77 \pm 0.33$ & $2.36 \pm 0.19$ \\
\hline
\end{tabular}

Spontaneous baroreflex parameters of transgenic animals as shown in Table 2 but now calculated from $2 \mathrm{~h}$ recordings at rest.

\section{Discussion}

In the present study, transgenic rats overexpressing TPM1 with one of the two disease-causing mutations Asp175Asn and Glu180Gly were studied for changes in HRV, BPV, and BRS. The data show that the analysis of cardiac autonomic control (particularly of baroreflex regulation) by using advanced signal processing tools represents a sensitive approach to discriminate between the effects of point mutations within a single sarcomeric protein on cardiac function in vivo.

Changes of HRV parameters were detected in transgenic rats with either mutation Asp175Asn or Glu180Gly in TPM1 compared to transgenic controls. During exercise, in both mutant lines, B (Asp175Asn) and C (Glu180Gly), increased LF power and elevated LF/HF ratio were observed (Table 1). At the same time, for line B (Asp175Asn) but not for line C (Glu180Gly), a decrease of total HRV was shown, which is known to be associated with a poor prognosis in patients with different heart diseases [11, 16]. Compared with mutation Glu180Gly, mutation Asp175Asn represents a so-called "hot spot" mutation of the human disease, which was detected in five unrelated families $[6,22]$ and shows a more severe clinical phenotype [18]. Accordingly, in our previous study, transgenic rats with mutation Asp175Asn demonstrated pronounced alterations in cardiac physiology than animals with mutation Glu180Gly [22]: Only cardiac skinned-fiber preparations of line B (Asp175Asn) demonstrated reduced $\mathrm{Ca}^{2+}$ sensitivity. In cardiomyocytes of line B (Asp175Asn), increased frequency and amplitude of spontaneous $\mathrm{Ca}^{2+}$ waves suggest an increase in intracellular $\mathrm{Ca}^{2+}$ concentration compensating for the reduced $\mathrm{Ca}^{2+}$ sensitivity of isometric force generation. As a consequence, increased intracellular $\mathrm{Ca}^{2+}$ might lead to a "hypercontractile" state of the myofilament system in line B (Asp175Asn). Interestingly to note that FHC is usually accompanied with impaired left ventricular diastolic function and secondary left atrial overloading [3, 9]. Thus, modulation of atrial and ventricular stretchmediated heart rate control, such as the Bainbridge reflex, may be one of the factors responsible for both decreased HRV and alteration of autonomic nervous activity [8].
Further, the analysis of the spontaneous baroreflex activity revealed a strong influence of the baroreflex on $\mathrm{BP}$ regulation in the transgenic rats with either mutation Asp175Asn or Glu180Gly in TPM1 compared to transgenic controls. During exercise, in lines B (Asp175Asn) and C (Glu180Gly) a decreased BRS was accompanied by an increase in the number of bradycardic and tachycardic fluctuations. Furthermore, after completing the training protocol, resting rats of line B (Asp175Asn), but not line C (Glu180Gly), showed a strong increase in the number of tachycardic fluctuations (Table 3B). These data suggest frequent BP declines in animals with mutation Asp175Asn, known as to have a more severe phenotype in human [18] and to alter cardiac function in vitro to a higher extent than mutation Glu180Gly [22]. Frequent episodes of BP decline in line B (Asp175Asn) correspond well with data obtained from clinical studies with FHC patients. Exercise hypotension was observed in one-third of patients with FHC [13], due to an exaggerated paradoxic fall in systemic vascular resistance, postulated to be related to the activity of left ventricular mechanoreceptors [19]. Possibly, abnormal local left ventricular wall stresses during exercise and central hypovolemia may be responsible for left ventricular mechanoreceptor activation. Similar abnormal reflex responses were observed in patients with vasovagal syncope [20] and severe heart failure [1].

Sudden cardiac death is the most prominent feature of $\mathrm{FHC}$, although the relevant mechanisms are still incompletely understood. The data of the present study suggest that for mutation Asp175Asn and Glu180Gly in TPM1 several factors might trigger sudden cardiac death:

(i) It was shown that in $\mathrm{FHC}$ episodes of hypotension, particularly during or soon after exertion, are associated with an increased risk of sudden cardiac death [14]. The mechanism might be an abnormal behavior of the left ventricular mechanoreceptors during central blood volume underloading. To compensate for these alterations of BP regulation, strong baroreflex activity is needed as shown here for both mutant rat lines B (Asp175Asn) and C (Glu180Gly).

(ii) The increase in LF/HF ratio and LF power in lines $B$ (Asp175Asn) and C (Glu180Gly) can be considered 
as a reduced vagal activity and/or increased sympathetic activity known to be associated with lifethreatening arrhythmias during myocardial ischemia $[10,17]$. In parallel, in FHC patients who have survived cardiac arrest, electrophysiological testing has demonstrated that sinus node dysfunction and inducible ventricular arrhythmias are common [12].

(iii) In our previous study, it was shown that skinnedfiber preparations of line B (Asp175Asn) demonstrated reduced $\mathrm{Ca}^{2+}$ sensitivity, which is compensated by elevated intracellular $\mathrm{Ca}^{2+}$ concentration [22]. An increase in intracellular $\mathrm{Ca}^{2+}$ concentration could activate transient inward currents generally assumed to be arrhythmogenic [15]. Interestingly, mutations in thin filament proteins (TPM1, troponin T), in contrast to mutations in thick filament proteins ( $\beta$-myosin heavy chain, myosin-binding protein $C$ ), are characterized by a higher percentage of sudden cardiac death [2].

In summary, the analysis of the autonomic cardiac regulation represents a sensitive approach which may be able to discriminate between distinct FHC causing mutations within a single sarcomeric protein. In this context, the role of baroreflex regulation for early diagnosis and disease control in human has to be further investigated. In particular, clinical studies should be performed in cohorts with a defined genotype to investigate autonomic cardiac regulation for individual FHC causing mutations at rest and during exercise. Furthermore, the value of autonomic imbalance in predicting susceptibility to arrhythmias and sudden cardiac death and as a potential and independent marker of the disease should be addressed. The advanced signal processing tools used in this work proved to be very appropriate for such more extended research efforts.

\section{References}

[1] Atherton JJ, Thomson HL, Moore TD, et al. Diastolic ventricular interaction: a possible mechanism for abnormal vascular responses during volume unloading in heart failure. Circulation 1997; 96: 4273-4279.

[2] Bashyam MD, Savithri GR, Kumar MS, et al. Molecular genetics of familial hypertrophic cardiomyopathy (FHC). J Hum Genet 2003; 48: 55-64.

[3] Bonaduce D, Petretta M, Betocchi S, et al. Heart rate variability in patients with hypertrophic cardiomyopathy: association with clinical and echocardiographic features. Am Heart J 1997; 134: 165-172.

[4] Brockway BP, Mills PA, Azar SH. A new method for continuous chronic measurement and recording of blood pressure, heart rate and activity in the rat via radio-telemetry. Clin Exp Hypertens A 1991; 13: 885-895.

[5] Counihan PJ, Fei L, Bashir Y, et al. Assessment of heart rate variability in hypertrophic cardiomyopathy. Association with clinical and prognostic features. Circulation 1993; 88: 1682-1690.

[6] Coviello DA, Maron BJ, Spirito P, et al. Clinical features of hypertrophic cardiomyopathy caused by mutation of a "hot spot" in the alpha-tropomyosin gene. J Am Coll Cardiol 1997; 29: 635-640.

[7] Doven O, Sayin T, Guldal M, et al. Heart rate variability in hypertrophic obstructive cardiomyopathy: association with functional classification and left ventricular outflow gradients. Int J Cardiol 2001; 77: 281-286.

[8] Eleuteri E, Lanfranchi P, Scapellato F, et al. Restrictive left ventricular filling pattern as a strong predictor of depressed baroreflex sensitivity in heart failure. Ital Heart J 2001; 2: 344-348.

[9] Isobe N, Toyama T, Taniguchi K, et al. Failure to raise blood pressure during exercise is a poor prognostic sign in patients with hypertrophic non-obstructive cardiomyopathy. Circ J 2003; 67: 191-194.

[10] La Rovere MT, Pinna GD, Hohnloser SH, et al. Baroreflex sensitivity and heart rate variability in the identification of patients at risk for life-threatening arrhythmias: implications for clinical trials. Circulation 2001; 103: 2072-2077.

[11] Lombardi F, Mortara A. Heart rate variability and cardiac failure. Heart 1998; 80: 213-214.

[12] McKenna WJ, Sadoul N, Slade AK, et al. The prognostic significance of nonsustained ventricular tachycardia in hypertrophic cardiomyopathy. Circulation 1994; 90: 31153117.

[13] Olivotto I, Maron BJ, Montereggi A, et al. Prognostic value of systemic blood pressure response during exercise in a community-based patient population with hypertrophic cardiomyopathy. J Am Coll Cardiol 1999; 33: 2044-2051.

[14] Sadoul N, Prasad K, Elliott PM, et al. Prospective prognostic assessment of blood pressure response during exercise in patients with hypertrophic cardiomyopathy. Circulation 1997; 96: 2987-2991.

[15] Stuyvers BD, Boyden PA, ter Keurs HE. Calcium waves: physiological relevance in cardiac function. Circ Res 2000; 86: 1016-1018.

[16] Tapanainen JM, Thomsen PE, Kober L, et al. Fractal analysis of heart rate variability and mortality after an acute myocardial infarction. Am J Cardiol 2002; 90: 347-352.

[17] Task Force of the European Society of Cardiology and the North American Society of Pacing and Electrophysiology. Heart rate variability: standards of measurement, physiological interpretation and clinical use. Circulation 1996; 93 : 1043-1065.

[18] Thierfelder L, MacRae C, Watkins H, et al. A familial hypertrophic cardiomyopathy locus maps to chromosome $15 \mathrm{q} 2$. Proc Natl Acad Sci USA 1993; 90: 6270-6274.

[19] Thomson HL, Morris-Thurgood J, et al. Reduced cardiopulmonary baroreflex sensitivity in patients with hypertrophic cardiomyopathy. J Am Coll Cardiol 1998; 31: 13771382.

[20] Thomson HL, Wright K, Frenneaux M. Baroreflex sensitivity in patients with vasovagal syncope. Circulation 1997; 95: 395-400.

[21] Uemura S, Tomoda Y, Fujimoto S, et al. Heart rate variability and ventricular arrhythmia in clinically stable patients with hypertrophic cardiomyopathy. Jpn Circ J 1997; 61: 819-826.

[22] Wernicke D, Thiel C, Duja-Isac CM, et al. Alpha-tropomyosin mutations Asp (175) Asn and Glu (180) Gly affect cardiac function in transgenic rats in different ways. Am J Physiol Regul Integr Comp Physiol 2004; 287: R685-R695.

[23] Wessel N, Bauernschmitt R, Wernicke D, Kurths J, Malberg $\mathrm{H}$. Autonomic cardiac control in animal models of cardiovascular diseases. I. Methods of variability analysis. Biomed Tech 2007; 52: 43-49. 